\title{
Flow cytometric immunophenotyping of canine adipose-derived mesenchymal stem cells (ADMSCs) and feline ADMSCs using anti-human antibodies
}

\author{
Minho Ko ${ }^{1, \dagger}$, Kwon Young Lee ${ }^{2, \dagger}$, Sae Hoon Kim ${ }^{3}$, Manho Kim ${ }^{4}$, Jung Hoon Choi ${ }^{1}$, \\ Wooseok Im ${ }^{4, *}$, Jin Young Chung ${ }^{1, *}$ \\ ${ }^{1}$ Department of Veterinary Internal Medicine and Institute of Veterinary Science, and ${ }^{2}$ Department of Veterinary Anatomy, \\ College of Veterinary Medicine, Kangwon National University, Chuncheon 24341, Korea \\ Departments of ${ }^{3}$ Orthopedic Surgery, and ${ }^{4}$ Neurology, College of Medicine, Seoul National University Hospital, Seoul 03080, Korea
}

(Received: February 21, 2018; Revised: March 13, 2018; Accepted: March 22, 2018)

\begin{abstract}
Various trials have been conducted to develop therapies for serious untreatable diseases. Among these, those using stem cells have shown great promise, and adipose-derived mesenchymal stem cells (ADMSCs) are easier to obtain than other types of stem cells. Prior to clinical trials, characterization of ADMSCs with monoclonal antibodies should be performed. However, it is difficult to use species-specific antibodies for veterinarians. This study was conducted to confirm the panel of human antibodies applicable for use in immunophenotypic characterization of canine adipose-derived stem cells and feline ADMSCs extracted from subcutaneous adipose tissue collected during ovariohysterectomy. For flow cytometric immunophenotyping, the third passages of canine ADMSC and feline ADMSC and human CD31, CD34, CD42, CD44, CD62 and CD133 antibodies were used. Of these, CD133 reacted with canine cells $(3.74 \%)$ and feline cells $(1.34 \%)$. CD133 is known as a marker related with more primitive stem cell phenotype than other CD series. Because this human CD133 was not a species-specific antibody, accurate percentages of immunoreactivity were not confirmed. Nevertheless, the results of this study confirmed human CD133 as a meaningful marker in canine and feline ADMSCs.
\end{abstract}

Keywords: adipose-derived mesenchymal stem cell, cats, dogs, flow cytometric immunophenotyping, human antibodies

\section{Introduction}

Mesenchymal stem cells (MSCs) are defined as multipotent cells that have ability of self-renewal, long-term viability, and mutilineage potential. MSCs can regenerate into bone, cartilage, muscle, tendon, ligament, adipose, and stroma in vitro and in vivo. Because of these potencies of MSCs, they have been used for human and veterinary treatments [9].

MSCs have various origins, including the bone marrow, umbilical cord tissue, adipose tissue, tooth birth and peripheral blood [2]. When compared to other cell types, MSCs are abundant in adipose tissue, which the number of MSCs in adipose tissue have been shown to be up to 300 times than in bone marrow [9]. Adipose-derived MSCs (ADMSCs) also have the advantage of easy access and rapid expansion in vitro [3].

Although there have been many studies of the therapeutic uses of ADMSCs in dogs and horses [9], few studies have investigated the use of ADMSCs in cats [10, 15], despite the high number of cats as companion animals.

Immunophenotyping is generally used to characterize ADMSCs in different animal species. However, immunophenotyping has been limited in veterinary medicine because of the lack of antibodies for the surface profile in some of the species [9]. To overcome these limitations, cross-reactive antibodies have been used; however, few studies have been conducted using cross-reactive antibodies [5]. Therefore, in this study, flow cytometric immunophenotyping of canine adipose and feline ADMSCs was conducted using antihuman antibodies.

\section{Materials and Methods}

\section{Animals}

Two beagle intact female dogs and two mixed breed intact female cats were used. Animals were determined to be healthy by physical examination. All animals underwent rou-

\footnotetext{
*Corresponding author

Tel: +82-33-250-8656, Fax: +82-33-259-5625

E-mails: imwooseok@gmail.com (W Im), jychung77@gmail.com (JY Chung)

The first two authors contributed equally to this work.
} 
tine ovariohysterectomy, under the situation of sedation with midazolam $(0.1 \mathrm{mg} / \mathrm{kg})$ and acepromazine $(0.03 \mathrm{mg} / \mathrm{kg})$ and then anesthetization with isoflurane $(0.25-3 \%$ isoflurane in $100 \%$ oxygen in an induction chamber). During ovariohysterectomy, 2-4 g of subcutaneous fat in ventral midline abdomen of each animal was obtained [10]. This study was approved by the Kangwon National University Institutional Care and Animal Use Committee (KW-160809-1).

\section{Isolation and expansion of ADMSCs}

Subcutaneous fat $(2-4 \mathrm{~g})$ in ventral midline abdomen of each animal was collected in phosphate buffered saline (PBS; Gibco, USA) during ovariohysterectomy. Each tissue sample was cut into small pieces and incubated in $0.075 \%$ of Collagenase I (Gibco) in HBSS (Sigma-Aldrich, USA) for $1 \mathrm{~h}$ at $37^{\circ} \mathrm{C}$ under $5 \% \mathrm{CO}_{2}$. After then the suspension including cells was neutralized with $10 \%$ fetal bovine serum (FBS; Gibco) and then centrifuged at $1,200 \times \mathrm{g}$ for $10 \mathrm{~min}$ at room temperature. The supernatant was then eliminated and the sediment was mixed with $5 \mathrm{~mL}$ RBC lysis buffer (Sigma-Aldrich) for $10 \mathrm{~min}$. Next, the suspension including cells got out a $100 \mu \mathrm{m}$ cell strainer (Flacon; Corning Life Sciences, USA) to remove excess tissue stroma and diluted with $10 \mathrm{~mL}$ PBS, after which it was centrifuged at $1,200 \times \mathrm{g}$ for $10 \mathrm{~min}$ at room temperature. The supernatant was then eliminated and the sediment was resuspended in $5 \mathrm{~mL}$ Dulbecco's Modified
Eagle Medium (Gibco) with 5\% FBS and plated in T-25 plastic tissue culture flasks. After the confluency of cells reached $80 \%$, cells were detached with $0.05 \%$ trypsin (Gibco). Then the cells were resuspended in $20 \mathrm{~mL}$ of medium and split into 2 T-75 plastic tissue culture flasks. After this, all cell cultures were passaged along the similar lines until the third passage of cells was available for characterization [10].

\section{Flow cytometric immunophenotyping}

Flow cytometry analysis was conducted to analyze the phenotypes of ADMSCs. Briefly, ADMSCs derived from dogs or cats were collected, washed with PBS, and stained for $1 \mathrm{~h}$ with antibodies. The specific antibodies used were phycoerythrin-conjugated mouse anti-human CD31, fluorescein isothiocyanate (FITC) conjugated mouse anti-human CD42b, FITC-conjugated mouse anti-human CD44, FITC-conjugated mouse anti-human CD62b, FITC-conjugated mouse anti-human CD133 (BD, USA), and FITC-conjugated mouse anti-human CD34 (Santa Cruz Biotechnology, USA). The labeled ADMSCs were analyzed using a FACScalibur (BD Bioscience, USA), after which the data were analyzed with a FACS II flow cytometer (BD Biosciences).

\section{Results}

The cells of the third passage in each dogs and cats dis-
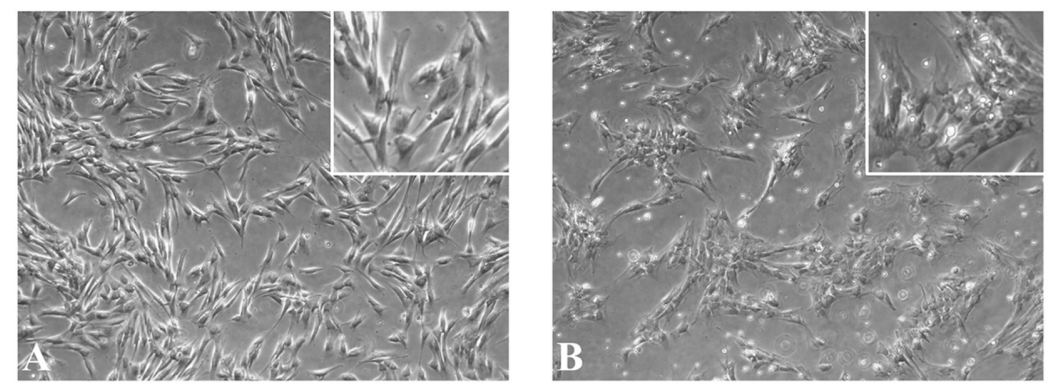

Fig. 1. The third passages of adipose-derived mesenchymal stem cells in dogs (A) and cats (B). $50 \times(A$ and B) and $100 \times($ insert).
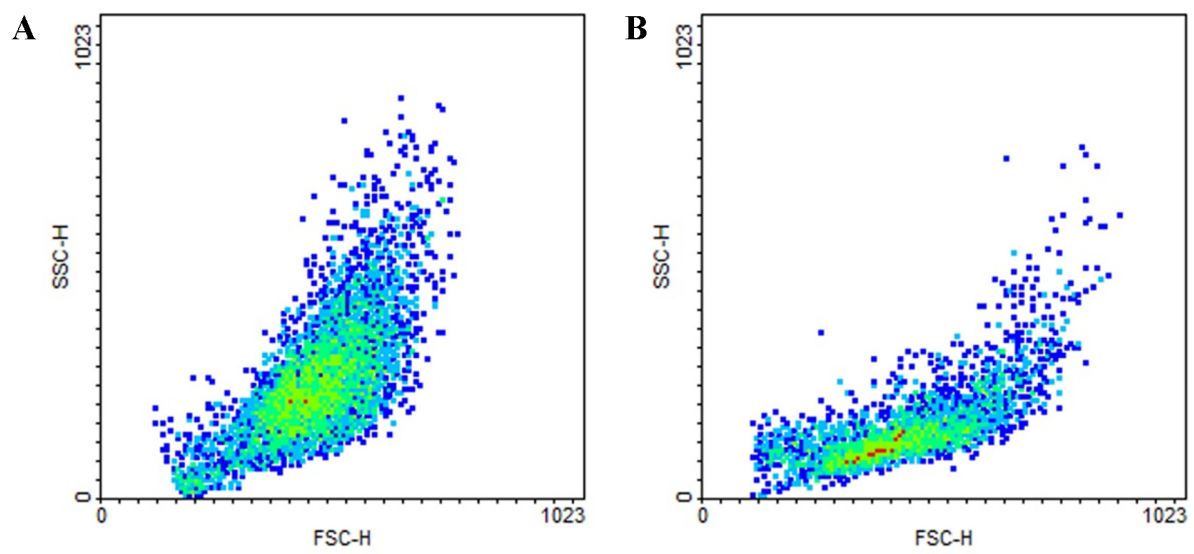

Fig. 2. Forward scatter $v s$. side scatter plots of adipose-derived mesenchymal stem cells (ADMSCs) in dogs (A) and cats (B). One cellular subset was identified in each ADMSC of dogs and cats with forward scatter (FSC-H)/side scatter (SCC-H) dot plots. 
A

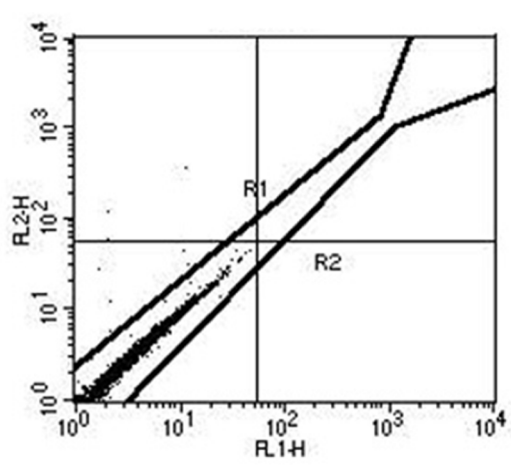

C

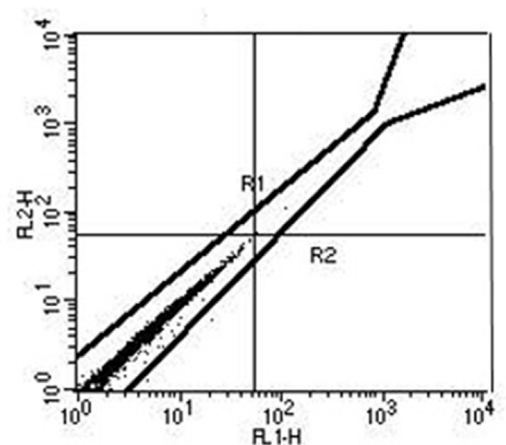

$\mathbf{E}$

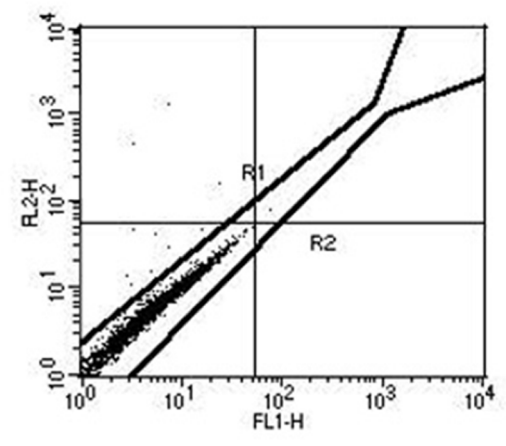

B

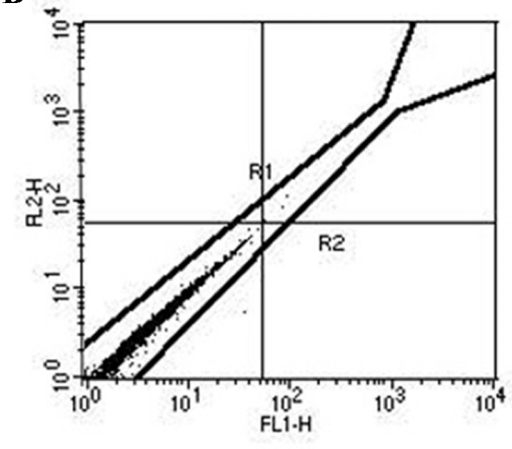

D

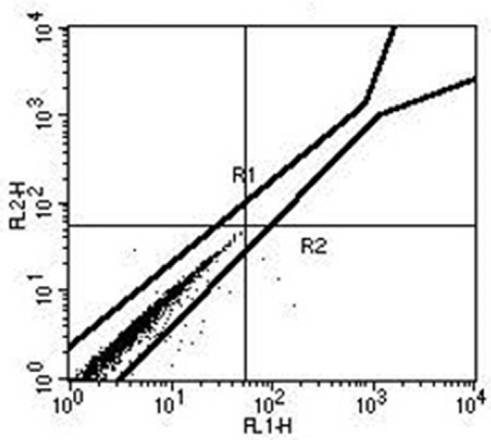

F

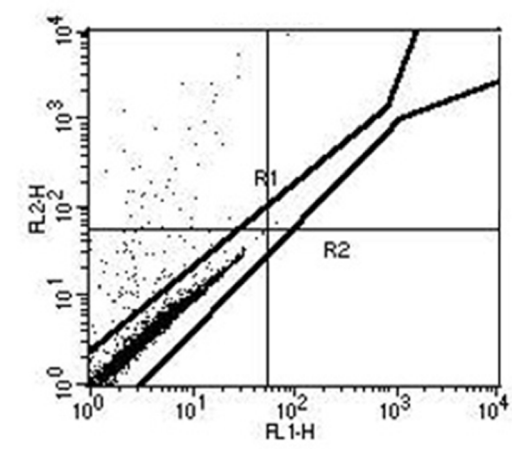

Fig. 3. Reactivity of the monoclonal antibodies in dogs. X-axis indicates the fluorescence intensity (FL1-FITC; FITC, fluorescein isothiocyanate) and Y-axis indicates the fluorescence intensity (FL2-PE; PE, phycoerythrin). This results revealed that the cells had cross-reactivity $0.15 \%, 0.1 \%, 0.07 \%, 0.73 \%, 0.38 \%$ and $3.74 \%$ with CD31 (A), CD34 (B), CD42 (C), CD44 (D), CD62 (E), and CD133 (F).

played the exacted MSC phenotype. The morphology of ADMSCs in dogs was more unified than in cats (Fig. 1).

After labelling ADMSCs with monoclonal antibodies, the scatter-plot profile was determined by displaying as the forward scatter (FSC-H) vs. side scatter (SSC-H). One cellular subset was identified in each ADMSC of dogs and cats (Fig. 2 ). The results revealed that each set of cultured cells was of one type. To test the cross-reactivity, six monoclonal antibodies against various $\mathrm{CD}$ molecules were tested in each ADMSC. Flow cytometric analysis of dog ADMSCs revealed that the cells had cross-reactivity $0.73 \%$ with CD44 and $3.74 \%$ with CD133, while it was $0.15 \%, 0.1 \%, 0.07 \%$ and $0.38 \%$ for CD31, CD34, CD42, and CD62, respectively (Fig. 3). Flow cytometric analysis of cat ADMSCs revealed that the cells had cross-reactivity or $0.62 \%, 0.68 \%, 1.34 \%, 0.05 \%, 0.07 \%$ and $0.12 \%$ with CD44, CD62 CD133, CD31, CD34, and CD42, respectively (Fig. 4).

\section{Discussion}

A great deal of attention has been focused on ADMSCs due to their potential ability as cell therapy products. In human medicine, beyond the basic study of ADMSCs, the massive production and banking of clinical applicable phase cells, issues related with legal regulations, of cell therapy in different countries currently registered are being aligned. [8, 9]. These phenomena are not relevant to human medicine, but also apply to veterinary medicine. In equine veterinary 
A

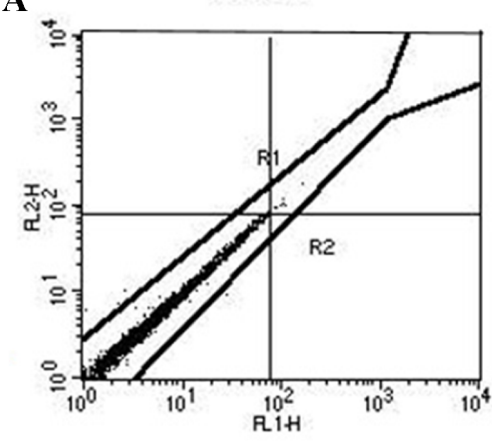

C

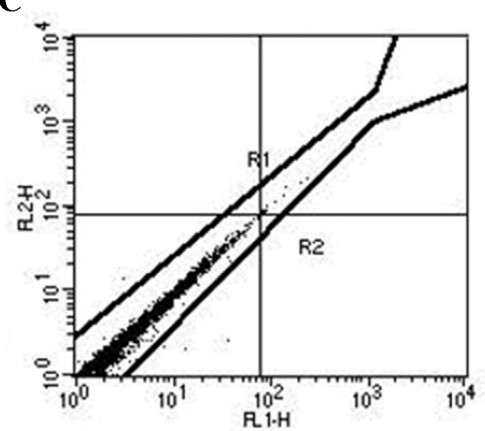

$\mathbf{E}$

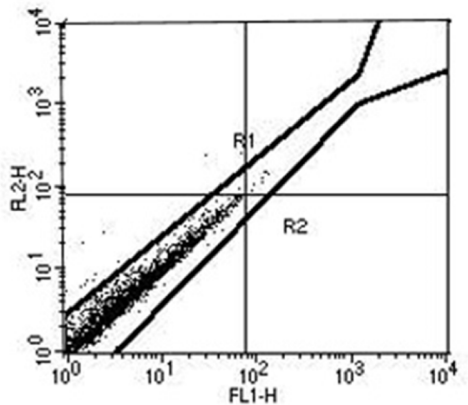

B

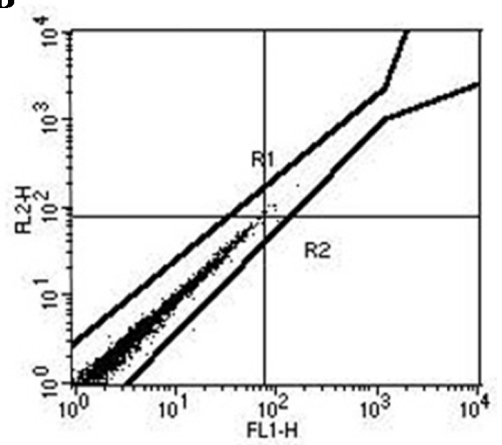

D

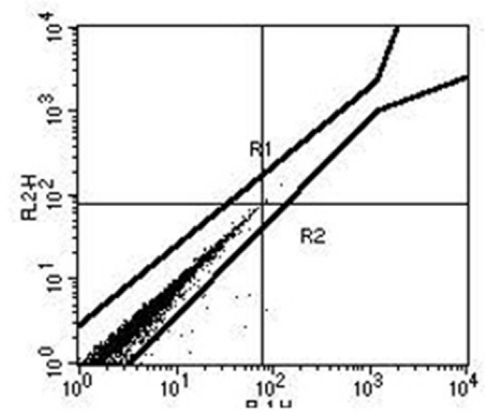

F

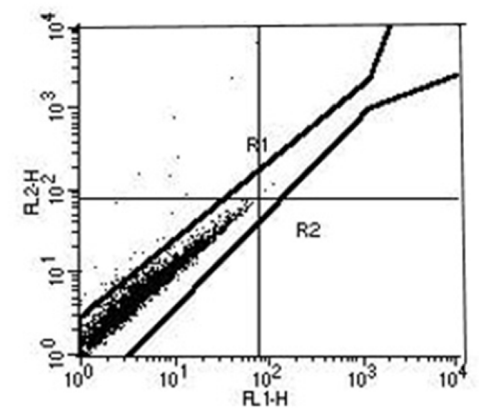

Fig. 4. Reactivity of the monoclonal antibodies in cats. X-axis indicates the fluorescence intensity (FL1-FITC) and Y-axis indicates the fluorescence intensity (FL2-PE). This results revealed that the cells had cross-reactivity $0.05 \%, 0.07 \%, 0.12 \%, 0.62 \%, 0.68 \%$ and $1.34 \%$, with CD31 (A), CD34 (B), CD42 (C), CD44 (D), CD62 (E), and CD133 (F).

medicine, clinical trials of ADMSCs have focused on tendon regeneration, while in small animal veterinary medicine, trials have focused on osteoarthritis and atopic dermatitis [1].

In human medicine, ADMSCs are confirmed based on cells being positive for CD13, CD73, CD90 and CD105 and negative for CD31, CD45, and CD235a upon immunophenotyping [4]. Despite the availability of ADMSCs, the use of species-specific antibodies in veterinary medicine in limited. Indeed, there have been few veterinary studies of immunophenotyping of ADMSCs. A recent study of immunophenotype showed that canine ADMSCs are positive for CD44, CD90 and MHC I, but negative for CD14, CD20 and MHC II [14]. Equine ADMSCs were shown to be positive for CD29, CD105, CD44, CD90, CD140b, and CD164 and negative for CD34, CD45, CD73 and MHC II [11]. Interestingly, other groups obtained conflicting results, with one group finding equine ADMSCs to be negative for CD44 and CD105 and the other group finding them to be both positive and negative for CD90 and CD34 $[12,13]$. In a study of feline ADMSCs, immunophenotyping showed that the cells were positive for CD44, CD90, and CD105, but negative for CD14, CD34 and CD45 [7]. As mentioned above, the opinions regarding immunophenotyping of ADMSCs in veterinary medicine are controversial. This is especially true in feline medicine because very few studies have investigated feline ADMSCs.

In this study, to test the possibility of other CDs, human CD31, CD34, CD42, CD44, CD62 and CD133 antibodies were analyzed by flow cytometric immunophenotyping. Among these, CD133 reacted with canine cells (3.74\%) and feline cells $(1.34 \%)$. CD133 positive cells are considered to have ability of early progenitors as self-renewing and differentiat- 
ing into other cell types. Therefore, CD133 appears to be a marker of primitive stem cell phenotype [6].

The human CD133 in this study was not species-specific; therefore, percentage was not confirmed. Nevertheless, the results of this study confirmed that CD133 is a meaningful marker associated with primate stem cells in canine and feline ADMSCs.

\section{Acknowledgments}

This work was supported by Basic Science Research Program through the National Research Foundation of Korea (NRF) funded by the Ministry of Science, ICT \& Future Planning (NRF-2017R1A1A1A05000762) and 2017 Research Grant from Kangwon National University (520170384).

\section{References}

1. Arnhold S, Wenisch S. Adipose tissue derived mesenchymal stem cells for musculoskeletal repair in veterinary medicine. Am J Stem Cells 2015, 4, 1-12.

2. Baer PC. Adipose-derived mesenchymal stromal/stem cells: an update on their phenotype in vivo and in vitro. World $\mathrm{J}$ Stem Cells 2014, 6, 256-265.

3. Bertassoli BM, de Assis Neto AC, de Oliveira FD, Arroyo MAM, Ferrao JSP, da Silva JB, Pignatari GC, Braga PB. Mesenchymal stem cells - emphasis in adipose tissue. Braz Arch Biol Technol 2013, 56, 607-618.

4. Bourin P, Bunnell BA, Casteilla L, Dominici M, Katz AJ, March KL, Redl H, Rubin JP, Yoshimura K, Gimble JM. Stromal cells from the adipose tissue-derived stromal vascular fraction and culture expanded adipose tissue-derived stromal/ stem cells: a joint statement of the International Federation for Adipose Therapeutics and Science (IFATS) and the International Society for Cellular Therapy (ISCT). Cytotherapy 2013, 15, 641-648.

5. Carrade DD, Borjesson DL. Immunomodulation by mesenchymal stem cells in veterinary species. Comp Med 2013, 63, 207-217.

6. Handgretinger R, Gordon PR, Leimig T, Chen $X$, Bühring HJ, Niethammer D, Kuçi S. Biology and plasticity of $\mathrm{CD}_{133^{+}}$hematopoietic stem cells. Ann N Y Acad Sci 2003, 996, 141-151.

7. Kono S, Kazama T, Kano K, Harada K, Uechi M, Matsumoto T. Phenotypic and functional properties of feline dedifferentiated fat cells and adipose-derived stem cells. Vet $\mathbf{J}$ 2014, 199, 88-96.

8. Lim MH, Ong WK, Sugii S. The current landscape of adipose-derived stem cells in clinical applications. Expert Rev Mol Med 2014, 16, e8.

9. Marx C, Silveira MD, Beyer Nardi N. Adipose-derived stem cells in veterinary medicine: characterization and therapeutic applications. Stem Cells Dev 2015, 24, 803-813.

10. Parys M, Nelson N, Koehl K, Miller R, Kaneene JB, Kruger JM, Yuzbasiyan-Gurkan V. Safety of intraperitoneal injection of adipose tissue-derived autologous mesenchymal stem cells in cats. J Vet Intern Med 2016, 30, 157-163.

11. Pascucci L, Curina G, Mercati F, Marini C, Dall'Aglio C, Paternesi B, Ceccarelli P. Flow cytometric characterization of culture expanded multipotent mesenchymal stromal cells (MSCs) from horse adipose tissue: towards the definition of minimal stemness criteria. Vet Immunol Immunopathol 2011, 144, 499-506.

12. Penny J, Harris P, Shakesheff KM, Mobasheri A. The biology of equine mesenchymal stem cells: phenotypic characterization, cell surface markers and multilineage differentiation. Front Biosci (Landmark Ed) 2012, 17, 892-908.

13. Ranera B, Lyahyai J, Romero A, Vázquez FJ, Remacha AR, Bernal ML, Zaragoza P, Rodellar C, Martín-Burriel I. Immunophenotype and gene expression profiles of cell surface markers of mesenchymal stem cells derived from equine bone marrow and adipose tissue. Vet Immunol Immunopathol 2011, 144, 147-154.

14. Screven R, Kenyon E, Myers MJ, Yancy HF, Skasko M, Boxer L, Bigley EC 3rd, Borjesson DL, Zhu M. Immunophenotype and gene expression profile of mesenchymal stem cells derived from canine adipose tissue and bone marrow. Vet Immunol Immunopathol 2014, 161, 21-31.

15. Trzil JE, Masseau I, Webb TL, Chang CH, Dodam JR, Liu H, Quimby JM, Dow SW, Reinero CR. Intravenous adipose-derived mesenchymal stem cell therapy for the treatment of feline asthma: a pilot study. J Feline Med Surg 2016, 18, 981-990. 